\section{Instrumentos de viento y su relación con el sistema estomatognático}

\section{Relationship between wind musical instruments and the stomatognatic system}

\section{Resumen}

Por la importancia que reviste ampliar los conocimientos del odontólogo en esta área, la presente revisión bibliográfica propone como objetivo resumir las modificaciones del sistema estomatognático que se presentan en músicos que practican instrumentos de viento. Dentro de las anomalías bucales que más abundan en la literatura se encuentran distonía focal, xerostomía, faringitis, queilitis angular, herpes labial y maloclusiones de una gran prevalencia e impacto, dentro de las más frecuentes registradas en este tipo de músicos se encuentran: el resalte aumentado, el apińamiento y la adaquia. El resalte aumentado es consecuencia de la presión que ejerce la boquilla en la cara palatina de los incisivos superiores, esto provoca que aumente la distancia entre la cara vestibular de los incisivos inferiores y la cara palatina de los incisivos superiores, en sentido anteroposterior (con las arcadas en oclusión). Se concluye que las presiones anormales que generan los instrumentos de viento sobre el aparato estomatognático, provocan una serie de alteraciones que pueden afectar la mucosa oral, los músculos implicados en la práctica instrumental y la oclusión dentaria.

Palabras clave: Cavidad bucal, Factores de riesgo; Maloclusiones.

\section{Artículo de Revisión}

Elizabeth de la Torre Rodríguez 1,a, Iris Aguirre Espinosa ${ }^{1, b}$, Jackeline Núñez Fernández ${ }^{1, b}$, Julio Danilo de la Torre Morales ${ }^{1, c}$, Camila León Ramis ${ }^{1, d}$

${ }^{1}$ Universidad de Ciencias Médicas de La Habana. Instituto Superior de Ciencias Básicas y Preclínicas Victoria de Girón. La Habana,

Cuba.

a Especialista en Anatomía Humana.

${ }^{\mathrm{b}}$ Especialista de Estomatología General Integral.

${ }^{c}$ Especialista en Ortodoncia.

${ }^{\mathrm{d}}$ Estudiante.

\section{Correspondencia:}

Elizabeth de la Torre Rodríguez

Correo electrónico: judani@infomed.sld.cu Freyre Andrade 220. Entre Mayía y Goicuría. La Habana, Cuba, código postal 10500.

Coautores:

Iris Aguirre Espinosa barnug@infomed.sld.cu Jackeline Núñez Fernández judani@infomed.sld.cu

Conflicto de intereses: Los autores declaran no tener conflictos de interés.

Fuente de financiamiento: Autofinanciado

Fecha de recepción: 30/06/18

Fecha de aceptación: 13/10/18 


\section{Introducción}

En la práctica de instrumentos de viento, los labios y los dientes se encuentran en estrecha relación con la boquilla ${ }^{1,2}$. En 1939, Strayer ${ }^{3}$, fagotista profesional y ortodoncista, fue el primero en plantear que la ejecución de instrumentos de viento alteraba la oclusión dentaria y por lo tanto producir o enmendar una maloclusión. En 1965, Engelman ${ }^{4}$ demostró que un instrumento de viento puede ejercer cinco veces más de fuerza de lo que se necesita para lograr un movimiento dentario.

Pang ${ }^{5}$ en 1976, concluyó que algunos instrumentos aumentan el resalte otros lo disminuyen y otros pueden producir mordida abierta anterior (adaquia). El Dr. Brattstrom ${ }^{6}$ en 1989 también investigó al respecto, en un estudio titulado Morfología Dentofacial en niños que tocan instrumentos de viento.

Recientemente, algunas investigaciones llevadas a cabo por Green et al. ${ }^{7}$, Stechman et al. ${ }^{8}$, Amores ${ }^{9}$ y Lavado et al. ${ }^{10}$ han estudiado la influencia de la práctica instrumental en la aparición de alteraciones del sistema estomatognático. Cuando la práctica instrumental se realiza durante extensas jornadas, las posturas perjudiciales se mantienen por largos periodos, lo cual propicia la aparición de alteraciones del sistema estomatognático ${ }^{11}$.

El Dr. Planas ${ }^{12}$ describió la rotura del músculo orbicular de los labios en trompetistas. Las dermatitis de contacto es otra de las alteraciones que se presenta, por el contacto de la piel con el instrumento. También se puede observar hiperqueratosis (engrosamiento de la capa externa de la piel) provocada por el contacto de la boquilla en mucosa de los labios ${ }^{13}$.

La práctica de instrumentos musicales es tomada en cuenta como un factor de riesgo de maloclusiones dentarias ${ }^{14}$. Por lo antes expuesto, reviste gran importancia incrementar los conocimientos del odontólogo sobre estos temas, pues el ejercicio del músico profesional actúa sobre su estado de salud, situación importante para que el odontólogo entienda algunas alteraciones funcionales y morfológicas de las estructuras del aparato estomatognático, además necesita visualizar los problemas potenciales a los cuales se enfrentan los músicos profesionales, de esta forma pueden ofrecer una asesoría preventiva y brindar un adecuado tratamiento rehabilitador que mejore su calidad de vida.

Surge entonces la siguiente interrogante: ¿Cuáles son las modificaciones del sistema estomatognático que se presentan en músicos que practican instrumentos de viento? La presente revisión plantea como objetivo resumir las modificaciones del sistema estomatognático que se presentan en músicos que practican instrumentos de viento.

\section{Desarrollo}

El sistema estomatognático se encuentra formado por un conjunto de estructuras esqueléticas, musculares, nerviosas, glandulares y dentales, su articulación principal es la articulación temporomandibular. El sistema estomatognático se utiliza como herramienta para la interpretación de instrumentos musicales ${ }^{15}$.

Rojas et al. ${ }^{16}$, en Colombia, estudiaron 13 músicos de instrumentos de viento, con una edad promedio de 25 años, alrededor de 15 años de experiencia como intérpretes y 19 horas semanales en la práctica. Durante el examen físico se observó que la maloclusión de mayor prevalencia fue el apiñamiento dental en un 38,46\%, con grado leve para todos los participantes en los que se presentó. Debido a que la mucosa oral es sometida a traumas constantes propios de la ejecución, se observó una prevalencia del 100\% de lesiones de tipo traumática en tejidos blandos. En casi la totalidad de la muestra se evidenció región hipertrófica a nivel de la mucosa yugal de forma bilateral. El examen clínico reveló la presencia en la mucosa del labio superior de una alteración hipertrófica de forma triangular, asintomática. La prevalencia de esta lesión fue del $76,92 \%$, junto a este hallazgo se encontró en un intérprete una fisura en labio superior. También se encontraron alteraciones neuromusculares tales como distonía focal. Otras de las alteraciones encontradas fueron: xerostomía, dolor en orofaringe, caries y gingivorragia en la mayoría de los examinados. Además, se evidenciaron ruidos en la articulación temporomandibular (ATM) en un 69\% de los músicos, estos constituyen signos clínicos de trastornos temporomandibulares (TTM), según el Índice de Helkimo, que fue modificado por Maglione ${ }^{17}$.

Por otra parte, uno de los factores predisponentes de los TTM son las maloclusiones y así lo refleja la investigación de Soto et al. ${ }^{18}$ la cual muestra que a medida que aumentó el número de maloclusiones en los pacientes estudiados, aumentó también la severidad de los TTM.

El complicado funcionamiento de la ATM determina que mínimas alteraciones produzcan en ella una gran diversidad de problemas funcionales ${ }^{19,20}$. Autores como Agudelo et al. ${ }^{21}$ han encontraron en sus investigaciones TTM con el aumento de la edad, sin embargo, otros como Meneses et al. ${ }^{22}$ y Aravena et al. ${ }^{23}$ demuestran la aparición de TTM en edades tempranas.

Mendes et al. ${ }^{24}$ en Argentina, estudiaron 20 músicos y encontraron un $100 \%$ de presencia de lesiones estomatológicas, entre las más frecuentes se encontraron: queilitis descamativa, queilitis fisurada y herpes labial recurrente. Amores et al. ${ }^{9}$ en Quito, Ecuador, estudiaron las maloclusiones en clarinetistas, como resultado encontraron que los estudiantes mantuvieron la Clase molar I derecha e izquierda lo que significa que el tiempo no produce cambios a nivel molar, mientras que, en el sector anterior, se demostró aumento del resalte en un 83\% de estudiantes a largo plazo, y un 69,2\% de estudiantes presentaron sobrepase aumentado a largo plazo.

De la Torre et al. ${ }^{25}$ en Cuba estudiaron músicos de instrumentos de viento y hallaron una alta prevalencia de maloclusiones $(51,9 \%)$, fundamentalmente de resalte aumentado $(36,5 \%)$.

Las maloclusiones tienen un significativo impacto negativo en la salud oral relacionado con la calidad de vida, 
por la inconformidad psicológica que genera, una investigación realizada por Chen et al. ${ }^{26}$ en adultos jóvenes así lo demuestra.

En el "Tratado de Ortodoncia" de Mayoral 27 la maloclusión se define como una mal posición dentaria, y comprende todo aquello que se aleja de la oclusión normal. Por otra parte, la oclusión se define como las relaciones entre los arcos dentarios ${ }^{28}$. En estos músicos las maloclusiones son provocadas por la colocación de la boquilla entre las arcadas ${ }^{23}$.

La mordida abierta anterior (adaquia) se caracteriza por la existencia de un espacio entre las superficies incisales de ambas arcadas ${ }^{27}$. La sobremordida vertical u overbite, se define como la cantidad de incisivos inferiores que es cubierta por los incisivos superiores en su cara vestibular. Normalmente, se mide en tercios $(1 / 3,2 / 3,3 / 3$ y 0 o borde a borde), aunque también se puede medir en milímetros ${ }^{28}$.

Tres de los artículos revisados 9,16,25, identificaron las maloclusiones de mayor prevalencia en la población estudiada (Tabla).

Tabla. Maloclusión de mayor prevalencia en músicos

\begin{tabular}{llc}
\hline Estudio & $\begin{array}{c}\text { Maloclusión de mayor } \\
\text { prevalencia }\end{array}$ & $\begin{array}{c}\text { Porcentaje de músicos } \\
\text { afectados }\end{array}$ \\
\hline Amores $^{9}$ & Resalte aumentado & $83 \%$ \\
Rojas et al. ${ }^{16}$ & Apiñamiento dental & $38,46 \%$ \\
De la Torre et al. ${ }^{25}$ & Resalte aumentado & $36,5 \%$ \\
\hline
\end{tabular}

El resalte aumentado es consecuencia de la presión que ejerce la boquilla de instrumentos como el clarinete y el saxofón en la cara palatina de los incisivos superiores, esto provoca que aumente la distancia desde la cara vestibular de los incisivos inferiores hasta la cara palatina de los incisivos superiores, en sentido anteroposterior (con las arcadas en oclusión) ${ }^{25}$.

El resalte aumentado horizontal aumentado provoca afectaciones psicológicas serias. En un estudio realizado por Fabian et al. ${ }^{29}$ se demostró que los niños y adolescentes que presentan un resalte $>6 \mathrm{~mm}$, manifiestan limitaciones significativas en cuanto a la autopercepción de su salud bucal relacionada con la calidad de vida, en comparación a los niños que tienen un resalte normal (2-3 mm), sin embargo, el sobrepase aumentado y la adaquia, tuvieron una pequeña influencia en los pacientes estudiados. Resultados similares arroja el estudio realizado por Sierwald et $a l^{30}$.

El odontólogo en promoción de salud debe enfatizar en que los estudiantes principiantes no adquieran malas costumbres, deficiencias y problemas innecesarios en la práctica del instrumento, que son muy difíciles de resolver en años de madurez ${ }^{24}$.

\section{Conclusiones}

Se concluye que las presiones anormales que generan los instrumentos de viento sobre el aparato estomatog- nático, provocan una serie de alteraciones que pueden afectar la mucosa oral, los músculos implicados en la práctica instrumental y la oclusión dentaria.

\section{Referencias bibliográficas}

1. Queiroz JR, Mollica FB, Benetti P, de Araujo MA, Valera MC. Degree of chronic orofacial pain associated to the practice of musical instruments in orchestra's participants. Indian J. Dent Res. 2014;25(1):2831-7.

2. Katarzyna B, Iulian K, Dorota O. Wind Instruments and their Influence on Oral Cavity: Systematic Review. World J Dent. 2014;5(3):180-3.

3. Strayer ER. Musical instruments as an aid in the muscle defects and perversions. Angle Orthodontist. $1939 ; 9(2): 18-27$

4. Engelman JA. Measurement of perioral pressures during the playing of musical wind instruments. Am J Orthodontics. 1965;51(11):856-63.

5. Pang A. Relation of musical instruments to malocclusion. J Am Orthodontics. 1972; 92:565-70.

6. Battstrom V, Odenrick L, Kvam E. Dentofacial morphology in children playing musical wind instruments: a longitudinal study. Eur J Orthodontics. 1989;11:179-85.

7. Green H, Green S. The interrelationship of wind instrument technic, orthodontic treatment, and orofacial myology. IntJ Orafacial Myology. 1999;25:18-29.

8. Stechman J, de Almeida C, Rodríguez E, Bornia JC, Silvério $\mathrm{KC}$, de Aguiar MM, et al. Ocurrencia de signos y síntomas de disfunción temporomandibulares en músicos. Rev Soc Bras Fonoaudiol [Internet] 2009 [citado 3 enero 2018]; 14 (3) Disponible en: http://www.scielo. br/scielo.php?pid=S1516-80342009000300012\&scrip$\mathrm{t}=\mathrm{sci}$ arttext\&tlng=en

9. Amores FS. Maloclusiones dentarias en estudiantes que tocan el clarinete en el Conservatorio Nacional de Música Noviembre 2015 - Enero 2016. [Tesis de bachiller]. [Ecuador]: Universidad Central del Ecuador. Facultad de Odontología; 2016. 69 p.

10. Lavado JL, Limaymanta JM. Trastornos temporomandibulares en músicos intérpretes de instrumentos de viento metal - madera - Huancayo. [Tesis de bachiller]. [Perú]: Universidad de Huancayo; 2017. 112 p.

11. Hattori M, Sumita YI, Taniguchi H. Influence of changes in the oral cavity on the performance of recorder players: A pilot study. J Prosthet Dent. 2014;111(5):42529.

12. Planas J. Further experience with rupture of the orbicularis oris in trumpet players. Plast Reconstr Surg. 1988;81(6):975-81.

13. Rueda SJ, Roa JA, Montaguth C, Quintero J, Rivera M. Prevención de lesiones orales en tejidos blandos en Instrumentistas de viento mediante el uso de aparatos bucales removible [Tesis de bachiller]. [Colombia]: Universidad Santo Tomás; 2015.32 p

14. Mendez J, Morinelly J, Rojas D. Lesiones en sistema estomatognático asociado a la Interpretación de Instrumentos musicales. Una revisión de literatura. [Tesis de bachiller]. [Colombia]: Universidad Santo Tomás; 2016. 23 p. 
15. Montero JM, Leonora da Conceição L, Semykina O. La oclusión dentaria en interacción con la postura corporal. Rev Cubana Estomatol. [Internet] 2014 [citado 3 enero 2018];51(1). Disponible en: http://scielo.sld.cu/scielo. php?pid=S0034-75072014000100003\&script=sci_art text\&tlng=pt

16. Rojas AM, Lobo LA, De la Hoz R, Llinás A. Lesiones estomatológicas por el uso de instrumentos de viento en sus ejecutantes. Rev Ciencia Salud Virtual. [Internet] 2017[citado 3 enero 2018];9 (2): 67-77. Disponible en: http://revistas.curn.edu.co/index.php/cienciaysalud/article/view/974/848

17. Tabla Índice de Helkimo Modificada por Maglione. [Internet]. Universidad Nacional de Trujillo Facultad de Estomatología [citado 3 enero 2018]. Disponible en: https://es.scribd.com/document/363342427/Tabla-Indice-de-Helkimo-Modif-PorMaglione-Avch-Final

18. Soto L, de la Torre D, Aguirre I, de la Torre E. Trastornos temporomandibulares en pacientes con maloclusiones. Rev Cubana Estomatol. [Internet] 2013 [citado 3 enero 2018];50(4). Disponible en: http://scielo.sld.cu/scielo. php?pid=S003475072013000400005\&script=sci_arttext\&tlng=pt

19. Vásconez M, Bravo W, Villavicencio E. Factores asociados a los trastornos temporomandibulares en adultos de Cuenca, Ecuador. Rev Estomatol Herediana. [Internet] 2017 [citado 3 enero 2018]; 27(1). Disponible en: www. upch.edu.pe/vrinve/dugic/revistas/index.php/REH/article/download/3097/3046

20. Lambruschini V, Rimoldi M, Ruiz M, Beti M, Hernandez F, Levalle R, et al. Análisis comparativo de signos y síntomas asociados a los trastornos termporomandibulares a través de la aplicación del Índice de Fonseca y Helkimo modificado. Facultad de Odontología de La Plata. 2017 [citado 3 enero 2018]. Disponible en: http://sedici. unlp.edu.ar/bitstream/handle/10915/64046/Documento_completo.pdf-PDFA.pdf?sequence $=1$

21. Agudelo A, Vivares A, Posada A, Meneses J. Signos y síntomas de trastornos temporo-mandibulares en la población adulta mayor atendida en la red hospitalaria pública de Medellín (Colombia)._Rev Odontol Mex. 2016;20(3):193-01.

22. Meneses E, Vivares E, Martinez LI. Trastornos temporomandibulares y factores asociados en adolescentes y jóvenes de la ciudad de Medellín. [Internet] Rev Colomb Invetig Odontol. 2015 [citado 3 enero 2018]; 6(18). Disponible en: https:/www.rcio.org/index.php/rcio/article/view/215

23. Aravena PC, Arias R, Aravena R, Seguel F. Prevalencia de trastornos temporomandibulares en adolescentes del Sur de Chile, año 2015. Rev Clin Periodoncia Implantol Rehabil Oral. 2016;9:244-52.

24. Mendes C, Fernández R, Gamino A, Quevedo J, Doratti P, Barilaro L, et al. Músicos de viento: afecciones bucales asociadas a instrumentos musicales de viento. 2017. Facultad de Odontología de la Universidad Nacional de la Plata. [citado 3 enero 2018] Disponible en: http:// sedici.unlp.edu.ar/bitstream/handle/10915/65012/P\%C3\%B3ster.pdf-PDFA.pdf?sequence $=1$

25. de la Torre E, Aguirre I, de la Torre JD, Núñez J. Alteraciones estomatológicas en estudiantes de viola, violín e instrumentos de vientos Amadeo Roldán. Habana 2011. [Internet] Rev Haban Cienc Med. 2013[citado 3 enero 2018]; 12 (1). Disponible en: http://scielo.sld.cu/scielo.php?script=sci_arttext\&pi$\mathrm{d}=$ S1729-519X2013000100011

26. Chen M, Feng ZC, Liu X, Li ZM, Cai B, Wang DW. Impact of malocclusion on oral health-related quality of life in young adults. J Orofac Orthop. 2015; 85(6):986-91.

27. Mayoral J, Mayoral G, Mayoral P. Anomalías dentofaciales. En: Ortodoncia. Principios fundamentales y prácticas. La Habana: Editorial Científico Técnica; 1984. p. $127-68$

28. González G, Ardanza P. Rehabilitación protésica estomatológica. La Habana: Editorial Ciencias Médicas; 2008.

29. Fabian S, Gelbrich B, Hiemisch A, Kiess W, Hirsch C. Impact of overbite and overjet on oral health-related quality of life of children and adolescents. J Orofac Orthop. 2018;79(1):29-38

30. Sierwald I, John MT, Schierz O, Jost-Brinkmann P, Reissmann DR. Association of overjet and overbite with esthetic impairments of oral health-related quality of life. J Orofac Orthop. 2015;76(5):405-20. 